\title{
New Optimal Constant Weight Codes
}

\author{
I. Gashkov*, D. Taub \\ Department of Mathematics \\ Karlstad University, Sweden \\ igor.gachkov@kau.se, taub.math@gmail.com
}

Submitted: May 18, 2007; Accepted: Jun 18, 2007; Published: Jun 21, 2007

Mathematics Subject Classifications: 94B60

\begin{abstract}
In 2006, Smith et al. published a new table of constant weight codes, updating existing tables originally created by Brouwer et al. This paper improves upon these results by filling in 9 missing constant weight codes, all of which are optimal by the second Johnson bound. This completes the tables for $A(n, 16,9)$ and $A(n, 18,10)$ up to $n=63$ and corrects some $A(n, 14,8)$.
\end{abstract}

\section{Introduction}

A binary constant weight code is any subset of $\mathbb{B}^{n}$ such that all elements, codewords, have the same weight. An important problem in coding theory is finding $A(n, d, w)$, the maximum possible number of codewords in a constant weight code with length $n$, minimum distance $d$, and weight $w$.

A large table of lower bounds on these numbers was published by Brouwer et al. [1], and later added to by Smith et al. [4]. However, these tables are still far from complete, and many of the existing values can be improved upon.

When the lower bound for a code matches the upper bound, the code is said to be optimal. There are a number of different methods for determining upper bounds for constant weight codes, but for the codes presented in this paper the most useful is the one given by theorem 1 .

Theorem 1 (The second Johnson bound) There can be a code with parameters $n, d$ and $w$ and size $M$ only if

$$
(n-b) a(a-1)+b a(a+1) \leq\left(w-\left\lceil\frac{d}{2}\right\rceil\right) M(M-1)
$$

holds, where $a$ and $b$ are the unique integers such that $w M=a n+b$ and $0 \leq b<n$.

\footnotetext{
*The research was support by The Royal Swedish Academy of Sciences
} 
Proof See [2], p. 526.

The second Johnson bound $J_{2}(n, d, w)$ is the largest $M$ such that the inequality in theorem 1 holds. (It is possible that the inequality holds for all $M$, in which case $J_{2}(n, d, w)=\infty$.)

All of the codes presented in this paper are optimal by the second Johnson bound.

\section{New Lexicographic Methods}

In general, lexicographic methods, or lexicodes, rarely achieve good lower bounds. However, with some simple modifications, standard lexicodes can be used to obtain a number of useful results.

By adding a degree of randomness to a standard lexicode we were able to obtain a number of new optimal constant weight codes. Additional codes were found by using a genetic algorithm based on randomized lexicodes. These new codes complete the tables for $A(n, 16,9)$ and $A(n, 18,10)$ up to $n=63$.

A complete list of all new lower bounds, as well as the actual codes, is presented below. We presented binary vector $v=\left(b_{1}, b_{2}, \ldots, b_{n}\right)$ in support form $\left\{i \mid b_{i}=1\right\}$

\section{Results}

The results are given in two tables. All codes presented in this section are optimal by the second Johnson bound.

\section{Table 1}

\begin{tabular}{|c|c|c|c|c|c|c|c|c|c|}
\hline $\begin{array}{lll}\mathrm{n}: & 394041 \\
\mathrm{~A}(\mathrm{n}, 14,8): & 101011\end{array}$ & $\begin{array}{ll}2 & 43 \\
2 & 12\end{array}$ & & & & & & & & \\
\hline $\begin{array}{l}A(39,14,8)=10 \\
A(40,14,8)=10 \\
A(42,14,8)=12\end{array}$ & $\begin{array}{l}\text { ( } 4 \\
\text { he } \\
\text { he }\end{array}$ & $\begin{array}{l}\text { I4, } \\
\text { me } \\
\text { me }\end{array}$ & $\begin{array}{l}= \\
\mathrm{as} A \\
\mathrm{as} A\end{array}$ & $\begin{array}{l}1 \\
39 \\
41\end{array}$ & $\begin{array}{l}14 \\
14 \\
14\end{array}$ & 14 & 8) & $=12$ & $($ See $[6])$ \\
\hline & 1: & 1 & 2 & 3 & 4 & 5 & 6 & 7 & 8 \\
\hline & 2: & 1 & 9 & 10 & 11 & 12 & 13 & 14 & 15 \\
\hline & 3: & 1 & 16 & 17 & 18 & 19 & 20 & 21 & 22 \\
\hline & 4: & 23 & 2 & 9 & 16 & 32 & 33 & 34 & 35 \\
\hline & 5: & 23 & 3 & 10 & 17 & 28 & 29 & 30 & 31 \\
\hline & 6: & 23 & 4 & 11 & 18 & 24 & 25 & 26 & 27 \\
\hline 148$)=1$ & 7: & 5 & 12 & 19 & 24 & 28 & 32 & 36 & 37 \\
\hline & 8: & 6 & 13 & 20 & 33 & 29 & 25 & 36 & 38 \\
\hline & 9: & 7 & 14 & 21 & 34 & 30 & 26 & 37 & 39 \\
\hline & 10: & 8 & 15 & 22 & 35 & 31 & 27 & 39 & 38 \\
\hline & 11: & 38 & 30 & 12 & 4 & 16 & 40 & 41 & 42 \\
\hline & 12: & 36 & 31 & 7 & 18 & 9 & 40 & 43 & 44 \\
\hline & 13: & 39 & 32 & 20 & 3 & 11 & 41 & 43 & 45 \\
\hline & 14: & 37 & 25 & 22 & 2 & 10 & 42 & 44 & 45 \\
\hline
\end{tabular}




\section{Table 2}

n: $\quad 45464748485051525354555657585960616263$

A(n,16,9): 10101011111212131314151619192021222428

$A(45,16,9)=10($ See $[3])$

$A(46,16,9)=10$ and

$A(47,16,9)=10$ the same as $A(45,16,9)$.

$A(48,16,9)=11$ (See [5], p. 912-915.)

$A(49,16,9)=11$ the same as $A(48,16,9)$.

\begin{tabular}{|c|c|c|c|c|c|c|c|c|c|c|}
\hline \multirow{12}{*}{$A(50,16,9)=12$} & 1: & 1 & 2 & 3 & 4 & 5 & 6 & 7 & 8 & 9 \\
\hline & 2: & 1 & 10 & 11 & 12 & 13 & 14 & 15 & 16 & 17 \\
\hline & 3: & 2 & 10 & 18 & 19 & 20 & 21 & 22 & 23 & 24 \\
\hline & $4:$ & 3 & 11 & 18 & 25 & 26 & 27 & 28 & 29 & 30 \\
\hline & 5: & 4 & 12 & 19 & 25 & 31 & 32 & 33 & 34 & 35 \\
\hline & 6: & 5 & 13 & 20 & 26 & 31 & 36 & 37 & 38 & 39 \\
\hline & 7: & 6 & 14 & 21 & 27 & 32 & 36 & 40 & 45 & 50 \\
\hline & 8: & 7 & 15 & 18 & 31 & 40 & 41 & 42 & 43 & 44 \\
\hline & 9: & 8 & 16 & 22 & 25 & 37 & 42 & 45 & 46 & 47 \\
\hline & 10: & 5 & 17 & 23 & 29 & 33 & 43 & 45 & 48 & 49 \\
\hline & 11: & 1 & 24 & 28 & 34 & 38 & 44 & 46 & 48 & 50 \\
\hline & 12: & 9 & 10 & 30 & 35 & 39 & 41 & 47 & 49 & 50 \\
\hline
\end{tabular}

$A(51,16,9)=12$ the same as $A(50,16,9)$. See also [5], p. 912-915.

$\begin{array}{rrrrrrrrrrr}1: & 1 & 2 & 3 & 4 & 5 & 6 & 7 & 8 & 9 \\ & 2: & 1 & 10 & 11 & 12 & 13 & 14 & 15 & 16 & 17 \\ 3: & 2 & 10 & 18 & 19 & 20 & 21 & 22 & 23 & 24 \\ 4: & 3 & 11 & 18 & 25 & 26 & 27 & 28 & 29 & 30 \\ & 5: & 4 & 12 & 19 & 25 & 31 & 32 & 33 & 34 & 35 \\ & 6: & 5 & 13 & 20 & 26 & 31 & 36 & 37 & 38 & 39 \\ & 6: 52,16,9)=13 & 6 & 14 & 21 & 27 & 32 & 36 & 40 & 41 & 42 \\ & 7: & 7 & 15 & 22 & 28 & 33 & 37 & 40 & 47 & 52 \\ & 8: & 9 & 16 & 18 & 31 & 40 & 43 & 44 & 45 & 46 \\ 9: & 8 & 17 & 23 & 25 & 36 & 44 & 47 & 48 & 49 \\ 10: & 9 & 17 & 26 & 34 & 41 & 45 & 47 & 50 & 51 \\ 11: & 1 & 24 & 26 & 45 & 46 & 48 & 50 & 52 \\ 12: & 2 & 14 & 29 & 35 & 38 & 46 & \end{array}$

$A(53,16,9)=13$ the same as $A(52,16,9)$.

\begin{tabular}{|c|c|c|c|c|c|c|c|c|c|c|}
\hline & 1: & 1 & 2 & 3 & 4 & 5 & 6 & 7 & 8 & 9 \\
\hline & 2: & 1 & 10 & 11 & 12 & 13 & 14 & 15 & 16 & 17 \\
\hline & 3: & 2 & 10 & 18 & 19 & 20 & 21 & 22 & 23 & 24 \\
\hline & 4: & 3 & 11 & 18 & 25 & 26 & 27 & 28 & 29 & 30 \\
\hline & 5: & 4 & 12 & 19 & 25 & 31 & 32 & 33 & 34 & 35 \\
\hline & 6: & 5 & 13 & 20 & 26 & 31 & 36 & 37 & 38 & 39 \\
\hline \multirow{8}{*}{$A(54,16,9)=14$} & 7: & 6 & 14 & 21 & 27 & 32 & 36 & 40 & 41 & 42 \\
\hline & 8: & 7 & 15 & 22 & 28 & 33 & 37 & 40 & 43 & 44 \\
\hline & 9: & 8 & 16 & 23 & 29 & 34 & 38 & 41 & 43 & 46 \\
\hline & 10: & 1 & 18 & 31 & 42 & 44 & 45 & 46 & 47 & 48 \\
\hline & 11: & 9 & 17 & 19 & 26 & 40 & 46 & 49 & 50 & 51 \\
\hline & 12: & 3 & 13 & 24 & 32 & 43 & 47 & 49 & 52 & 53 \\
\hline & 13: & 2 & 15 & 25 & 39 & 41 & 48 & 50 & 52 & 54 \\
\hline & 14: & 7 & 10 & 27 & 35 & 38 & 45 & 51 & 53 & 54 \\
\hline
\end{tabular}




\begin{tabular}{|c|c|c|c|c|c|c|c|c|c|c|}
\hline & 1: & 1 & 2 & 3 & 4 & 5 & 6 & 7 & 8 & 9 \\
\hline & 2: & 1 & 10 & 11 & 12 & 13 & 14 & 15 & 16 & 17 \\
\hline & 3: & 2 & 10 & 18 & 19 & 20 & 21 & 22 & 23 & 24 \\
\hline & 4: & 3 & 11 & 18 & 25 & 26 & 27 & 28 & 29 & 30 \\
\hline & 5: & 4 & 12 & 19 & 25 & 31 & 32 & 33 & 34 & 35 \\
\hline & $6:$ & 5 & 13 & 20 & 26 & 31 & 36 & 37 & 38 & 39 \\
\hline & 7: & 6 & 14 & 21 & 27 & 32 & 36 & 40 & 41 & 42 \\
\hline$(55,16,9)=15$ & 8: & 7 & 15 & 22 & 28 & 33 & 37 & 40 & 43 & 44 \\
\hline & 9: & 8 & 16 & 23 & 29 & 34 & 38 & 41 & 43 & 45 \\
\hline & 10: & 9 & 17 & 24 & 30 & 35 & 39 & 42 & 44 & 45 \\
\hline & 11: & 1 & 18 & 31 & 40 & 45 & 46 & 47 & 48 & 49 \\
\hline & 12: & 2 & 11 & 32 & 38 & 44 & 47 & 50 & 51 & 52 \\
\hline & 13: & 3 & 10 & 35 & 36 & 43 & 48 & 50 & 53 & 54 \\
\hline & 14: & 4 & 16 & 22 & 26 & 42 & 49 & 51 & 53 & 55 \\
\hline & 15 & 6 & 12 & 23 & 28 & 39 & 46 & 52 & 54 & 55 \\
\hline
\end{tabular}

$A(56,16,9)=16($ See $[3])$

$A(57,16,9)=19($ See $[3])$

$A(58,16,9)=19$ the same as $A(57,16,9)$.

\begin{tabular}{|c|c|c|c|c|c|c|c|c|c|c|}
\hline & 1: & 1 & 2 & 3 & 4 & 5 & 6 & 7 & 8 & 9 \\
\hline & 2: & 1 & 10 & 11 & 12 & 13 & 14 & 15 & 16 & 17 \\
\hline & 3: & 1 & 18 & 19 & 20 & 21 & 22 & 23 & 24 & 25 \\
\hline & 4: & 1 & 26 & 27 & 28 & 29 & 30 & 31 & 32 & 33 \\
\hline & 5: & 34 & 6 & 14 & 22 & 30 & 35 & 36 & 37 & 38 \\
\hline & 6: & 34 & 7 & 15 & 23 & 31 & 39 & 40 & 41 & 42 \\
\hline & 7: & 34 & 8 & 16 & 24 & 32 & 43 & 44 & 45 & 46 \\
\hline & 8: & 34 & 9 & 17 & 25 & 33 & 47 & 48 & 49 & 50 \\
\hline & 9: & 51 & 2 & 10 & 18 & 26 & 38 & 42 & 46 & 50 \\
\hline \multirow{21}{*}{$A(59,16,9)=20$} & 10: & 51 & 3 & 11 & 19 & 27 & 37 & 41 & 45 & 49 \\
\hline & 11: & 51 & 4 & 12 & 20 & 28 & 36 & 40 & 44 & 48 \\
\hline & 12: & 51 & 5 & 13 & 21 & 29 & 35 & 39 & 43 & 47 \\
\hline & 13: & 5 & 12 & 45 & 50 & 23 & 30 & 52 & 53 & 54 \\
\hline & 14: & 19 & 26 & 35 & 40 & 9 & 16 & 52 & 55 & 56 \\
\hline & 15: & 4 & 13 & 38 & 41 & 25 & 32 & 52 & 57 & 58 \\
\hline & 16: & 18 & 27 & 44 & 47 & 7 & 14 & 53 & 56 & 8 \\
\hline & 17: & 2 & 20 & 37 & 43 & 15 & 33 & 53 & 57 & 89 \\
\hline & 18: & 11 & 29 & 42 & 48 & 6 & 24 & 54 & 57 & 55 \\
\hline & 19: & 3 & 21 & 36 & 46 & 17 & 31 & 54 & 56 & 59 \\
\hline & 20: & 10 & 28 & 39 & 49 & 8 & 22 & 55 & 58 & 59 \\
\hline & 1: & 1 & 2 & 3 & 4 & 5 & 6 & 7 & 8 & 9 \\
\hline & 2: & 1 & 10 & 11 & 12 & 13 & 14 & 15 & 16 & 17 \\
\hline & 3: & 2 & 11 & 18 & 19 & 20 & 21 & 22 & 23 & 24 \\
\hline & 4: & 2 & 10 & 25 & 26 & 27 & 28 & 29 & 30 & 31 \\
\hline & 5: & 3 & 10 & 18 & 32 & 33 & 34 & 35 & 36 & 37 \\
\hline & 6: & 1 & 18 & 25 & 38 & 39 & 40 & 41 & 42 & 43 \\
\hline & 7: & 4 & 11 & 25 & 33 & 44 & 45 & 46 & 47 & 48 \\
\hline & 8: & 2 & 13 & 32 & 39 & 44 & 49 & 50 & 51 & 52 \\
\hline & 9: & 3 & 12 & 19 & 26 & 38 & 44 & 53 & 54 & 55 \\
\hline & 10: & 3 & 11 & 29 & 39 & 56 & 57 & 58 & 59 & 60 \\
\hline \multirow{11}{*}{$A(60,16,9)=21$} & 11: & 5 & 14 & 22 & 27 & 32 & 40 & 46 & 53 & 56 \\
\hline & 12: & 5 & 12 & 21 & 28 & 34 & 41 & 47 & 50 & 57 \\
\hline & 13: & 9 & 12 & 20 & 27 & 36 & 42 & 45 & 49 & 58 \\
\hline & 14: & 6 & 14 & 20 & 26 & 34 & 43 & 48 & 51 & 59 \\
\hline & 15: & 6 & 15 & 22 & 28 & 33 & 42 & 52 & 54 & 60 \\
\hline & 16: & 8 & 13 & 24 & 28 & 35 & 40 & 45 & 55 & 59 \\
\hline & 17: & 9 & 16 & 19 & 30 & 35 & 41 & 46 & 51 & 60 \\
\hline & 18: & 4 & 17 & 21 & 30 & 36 & 43 & 52 & 55 & 56 \\
\hline & 19: & 7 & 16 & 24 & 27 & 37 & 38 & 48 & 52 & 57 \\
\hline & 20: & 7 & 15 & 23 & 29 & 35 & 43 & 47 & 49 & 53 \\
\hline & 21: & 8 & 17 & 23 & 31 & 37 & 46 & 50 & 54 & 58 \\
\hline
\end{tabular}




\begin{tabular}{|c|c|c|c|c|c|c|c|c|c|c|}
\hline & 1: & 1 & 2 & 3 & 4 & 5 & 6 & 7 & 8 & 9 \\
\hline & 2: & 1 & 10 & 11 & 12 & 13 & 14 & 15 & 16 & 17 \\
\hline & 3: & 2 & 10 & 18 & 19 & 20 & 21 & 22 & 23 & 24 \\
\hline & 4: & 1 & 20 & 25 & 26 & 27 & 28 & 29 & 30 & 31 \\
\hline & 5: & 1 & 18 & 32 & 33 & 34 & 35 & 36 & 37 & 38 \\
\hline & 6: & 2 & 12 & 25 & 32 & 39 & 40 & 41 & 42 & 43 \\
\hline & 7: & 4 & 11 & 18 & 26 & 39 & 44 & 45 & 46 & 47 \\
\hline & 8: & 5 & 10 & 26 & 34 & 40 & 48 & 49 & 50 & 51 \\
\hline & 9: & 3 & 10 & 25 & 33 & 46 & 52 & 53 & 54 & 55 \\
\hline & 10: & 2 & 11 & 28 & 33 & 48 & 56 & 57 & 58 & 59 \\
\hline \multirow{12}{*}{$A(61,16,9)=22$} & 11: & 3 & 13 & 19 & 27 & 34 & 39 & 56 & 60 & 61 \\
\hline & 12: & 9 & 12 & 19 & 29 & 36 & 45 & 49 & 52 & 57 \\
\hline & 13: & 5 & 15 & 22 & 27 & 35 & 41 & 44 & 52 & 59 \\
\hline & 14: & 9 & 13 & 20 & 35 & 42 & 47 & 51 & 53 & 58 \\
\hline & 15: & 17 & 22 & 28 & 38 & 42 & 45 & 50 & 54 & 61 \\
\hline & 16: & 8 & 12 & 21 & 30 & 37 & 44 & 48 & 53 & 60 \\
\hline & 17: & 7 & 17 & 23 & 29 & 32 & 44 & 51 & 55 & 56 \\
\hline & 18: & 4 & 14 & 20 & 36 & 43 & 50 & 55 & 59 & 60 \\
\hline & 19: & 8 & 14 & 23 & 31 & 33 & 41 & 47 & 49 & 61 \\
\hline & 20: & 6 & 16 & 21 & 27 & 38 & 43 & 46 & 51 & 57 \\
\hline & 21: & 6 & 15 & 24 & 30 & 36 & 40 & 47 & 54 & 56 \\
\hline & 22: & 7 & 16 & 24 & 31 & 37 & 39 & 50 & 52 & 8 \\
\hline
\end{tabular}

$A(62,16,9)=24$ and $A(63,16,9)=28($ See $[3])$.

\section{Table 2}

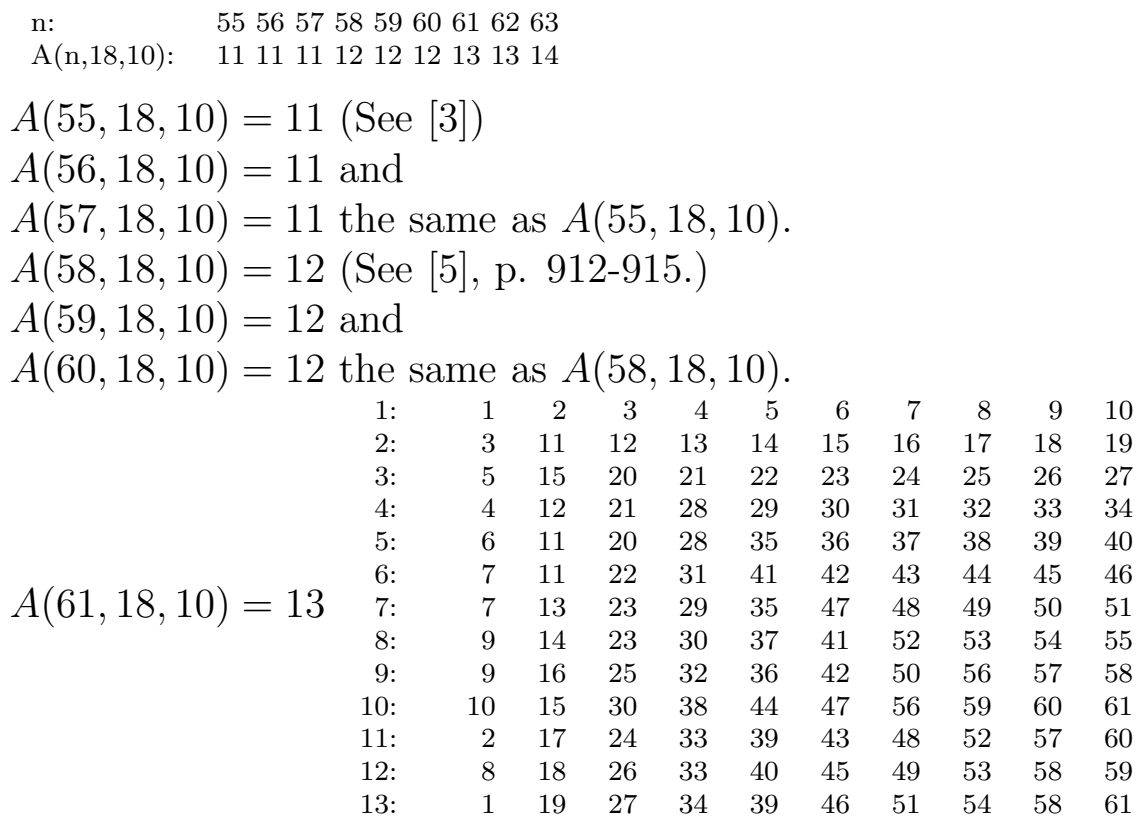

$A(62,18,10)=13$ the same as $A(61,18,10)$. 


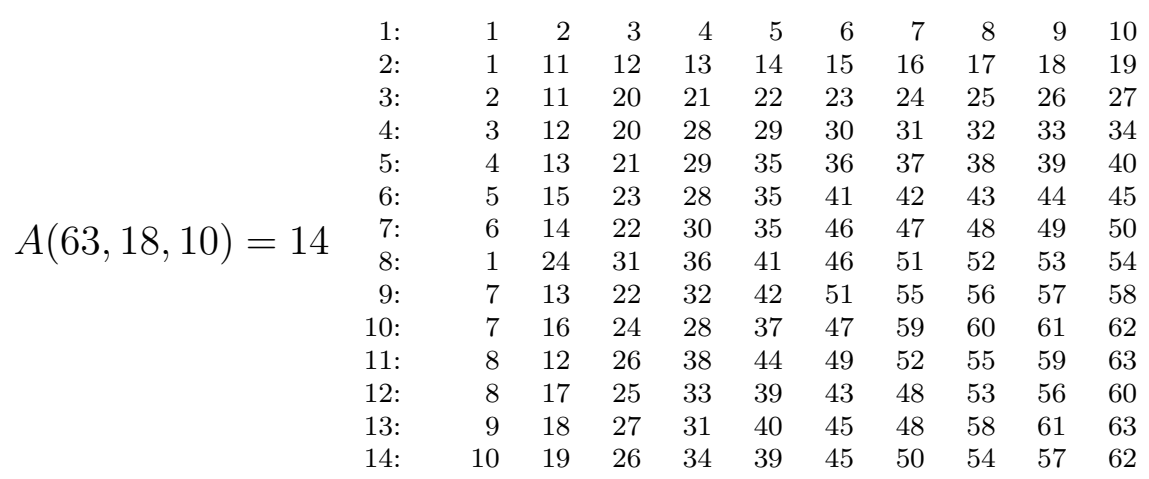

\section{References}

[1] A. E. Brouwer, J. B. Shearer, N. J. A. Sloane, W. D. Smith, "A New Table of Constant Weight Codes," IEEE Trans. Inform. Theory 36 (1990).

[2] F. J. MacWilliams, N. J. A. Sloane, The Theory of Error-Correcting Codes (NorthHolland, Amsterdam, 1979).

[3] E. M. Rains, N. J. A. Sloane, "Table of Constant Weight Binary Codes," http://www.research.att.com/ njas/codes/Andw/

[4] D. H. Smith, L. A. Hughes and S. Perkins, "A New Table of Constant Weight Codes of Length Greater than 28," Electron. J. Combin. 13 (2006).

[5] I. Gashkov, "Optimal Constant Weight Codes," Lecture note in Computer science, LNCS 3991 (2006).

[6] I. Gashkov, J.Ekberg, D.Taub, "A Geometric Approach to Finding New Lower Bounds of A (n, d, w)," Designs, Codes and Cryptography 43:2/3 June 2007. 